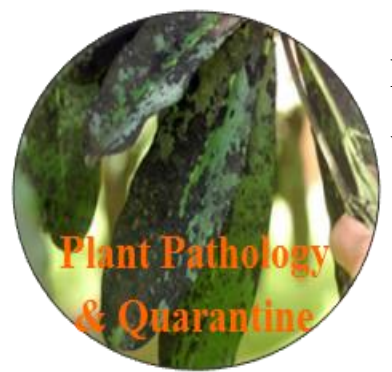

\title{
Are pathogenic isolates of Stemphylium host specific and cosmopolitan?
}

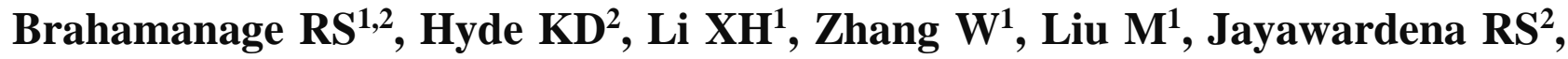 McKenzie EHC ${ }^{3,4}$ and Yan $\mathrm{JY}^{1 *}$}

${ }^{1}$ Institute of Plant and Environment Protection, Beijing Academy of Agriculture and Forestry Sciences, Beijing 100097, People's Republic of China

${ }^{2}$ Center of Excellence in Fungal Research, Mae Fah Luang University, Chiang Rai, 57100, Thailand

${ }^{3}$ Department of Biology, Faculty of Science, Chiang Mai University, Chiang Mai 50200, Thailand

${ }^{4}$ Manaaki Whenua - Landcare Research, Private Bag 92170, Auckland Mail Centre, Auckland 1142, New Zealand

Brahamanage RS, Hyde KD, Li XH, Jayawardena RS, McKenzie EHC, Yan JY 2018 - Are pathogenic isolates of Stemphylium host specific and cosmopolitan?. Plant Pathology \& Quarantine 8(2), 153-164, Doi 10.5943/ppq/8/2/7

\begin{abstract}
Stemphylium is a genus of filamentous ascomycetes comprising plant pathogens and saprobes in the family Pleosporaceae (Pleosporales, Dothideomycetes). Species of Stemphylium are known from a broad range of plant hosts including a variety of agricultural crops. This paper briefly discusses the occurrence of pathogenic isolates of Stemphylium on different host species, pathogenicity, disease severity, distribution and molecular phylogenetic affinities of pathogenic isolates of Stemphylium.
\end{abstract}

\section{Background}

Stemphylium Wallr. is a genus of filamentous ascomycetes comprising plant pathogens and saprobes in the family Pleosporaceae (Pleosporales, Dothideomycetes, Ascomycetes). The genus Stemphylium was proposed by Wallroth (1833) with S. botryosum Wallr. as the type species. About 200 published names currently represent recognizable taxa of Stemphylium (Woudenberg et al. 2017, Index Fungorum 2018). Stemphylium is a monophyletic genus, which comprises both saprophytic and plant pathogenic species with worldwide distribution (Köhl et al. 2009, Crous et al. 2016, Woudenberg et al. 2017). The genus can be distinguished from other dematiaceous hyphomycetes by its phaeodictyospores produced by the percurrent proliferation of its conidiophores, and by apically swollen conidiogenous cells (Crous et al. 2016, Woudenberg et al. 2017). The sexual morph of Stemphylium, is Pleospora which is a polyphyletic genus (Woudenberg et al. 2017). The type species of Pleospora, Pleospora herbarum, has Stemphylium herbarum as asexual morph (Simmons 1985), but several other Pleospora spp. have been linked to different asexual genera such as Alternaria, Coniothyrium and Bipolaris (Inderbitzin et al. 2009, De Gruyter et al. 2009, Ariyawansa et al. 2015, Crous \& Groenewald 2017). The use of Stemphylium over Pleospora has been recommended by the Working Group on Dothideomycetes of the International Committee on the Taxonomy of Fungi (Rossman et al. 2015). Stemphylium species are successful colonisers of decaying plant tissues (Hudson 1971, Köhl et al. 2009) and well known as plant pathogens (Falloon et al. 1987, Prados-Ligero et al. 1998, Basallote et al. 1993, Leuprecht 1990, Koike et al. 2001, Crous et al. 2016, Woudenberg et al. 2017). 


\section{Occurrence of pathogenic isolates of Stemphylium}

Stemphyium species have been reported from various agricultural crops such as, alfalfa, red clover, tomato and potato (Ellis \& Gibson 1975, Irwin 1984, Johnson \& Lunden 1986, Simmons 1990, Aveling \& Snyman 1993), sugar beet (Hanse 2013), asparagus, onion, garlic (Gálvez et al. 2016), birdsfoot trefoil (Lotus corniculatus) (Seaney 1973), pear, parsley, lentil, lucerne (Medicago sativa) and a variety of other horticultural crops (Falloon et al. 1987, Koike et al. 2013, Lamprecht et al. 1984, Llorente \& Montesinos 2006, Mentha 1998, Miller et al. 1978, Nasehi et al. 2013, Reis et al. 2011, Vakalounakis \& Markakis 2013, Subedi et al. 2014). Leaf spot disease accompanied by Stemphylium sp. was first discovered on sugar beet (Beta vulgaris) in the Netherlands, and later, it spread rapidly throughout the country (Hanse 2013). Crous et al. (2016) formally named the causal agent of this leaf spot disease as Stemphylium beticola. Stemphylium blight caused by S. botryosum is becoming a serious threat to lentil cultivation (Subedi et al. 2014) and has been reported in Bangladesh, Egypt, Nepal, Syria and the USA (Bayaa \& Erksine 1998). The causal agents of leaf spot in alfalfa and red clover are S. alfalfa, S. botryosum, S. globuliferum, S. herbarum, and $S$. vesicarium (Camara et al. 2002, Berg \& Leath 1996). Stemphylium vesicarium also causes purple spot in asparagus and leaf spot in onion and garlic (Gálvez et al. 2016). Stemphylium solani is the responsible pathogen for grey leaf spot on tomato and potato (Ellis \& Gibson 1975, Irwin 1984, Johnson \& Lunden 1986, Simmons 1990, Aveling \& Snyman 1993) Stemphylium loti has been reported as the causative agent for the most widespread foliar disease of birdsfoot trefoil (Seaney 1973). Stemphylium pathogens have been found from several vegetables and flowers, including asparagus (Asparagus officinalis), aster (Aster sp.), Chinese chives (Allium tuberosum), kalanchoe (Kalanchoe blossfeldiana), sweet pepper (Capsicum annuum), tomato (Lycopersicon esculentum), Welsh onion (Allium fistulosum), and white lace flower (Ammi majus) (Suzui 1973, Enjoji 1931, Ichikawa \& Sato 1994, Tomioka et al. 1997, Shibata et al. 2000, Misawa 2009, Kurose et al. 2015, Tomioka \& Sato 2011). Stemphylium brassicicola, S. ixeridis and S. microsporum have been reported from diseased leaves of Ixeris denticulata, Brassica pekinensis, and Malus sieversii, respectively, in Northwest China (Wang \& Zhang 2009). Two species of Stemphylium have been described as causing a rot of apple (Ruehle 1930). Stemphylium lycopersici isolates has been obtained from diseased tomato, eggplant (Solanum melongena), pepper and lettuce (Lactuca sativa) (Nasehi et al. 2014). Koike et al. (2001) reported that Stemphylium was causing leaf spot symptoms on spinach.

\section{Host specificity, pathogenicity, and disease severity}

There is lack of information on the relationship between saprophytic and pathogenic populations of Stemphylium spp. as well as on the possible host-specificity of pathogenic isolates (Köhl et al. 2009). However, Koike et al. (2001) demonstrated that isolates of S. botryosum from spinach appear to infect only spinach, and $S$. botryosum isolates from other sources do not infect spinach, thus showing host specificity. Mehta (2001) observed genetic difference between cotton and tomato isolates of $S$. solani causing leaf blight, hence providing evidence that $S$. solani attacking cotton in Brazil belongs to a distinct genotype. Leaf spot disease starts with small, irregular, yellow spots on the leaves of host species. The yellow spots become necrotic from the centre of the lesion outwards with tissue turning brown and the spots spread throughout all leaves of the plant. Ultimately, heavily infected leaves will die (Basallote-Ureba et al. 1999, Crous et al. 2016). Early symptoms of Stemphylium infection on garlic leaves appear as small white spots and apical necrosis. These lesions soon develop into larger, elongate white spots that eventually become purple and water soaked (Basallote et al. 1993). Fungal pseudothecia are able to persist on crop residues and ascospores are the primary inoculum in the following season (Basallote-Ureba et al. 1998, 1999). Once the disease is established, conidia form in primary lesions and rapidly disperse to infect healthy plants, resulting in a large decrease in photosynthesis and therefore in bulb yield reduction (Zheng et al. 2010). The occurrence of this disease drastically reduces garlic yield every year, around 70\%, in some fields in China (Zheng et al. 2010). Pseudothecia maturation and 
ascospore discharge are closely associated with high relative humidity and mild temperatures (Prados-Ligero et al. 1998).

\section{Distribution}

Both saprotrophic and pathogenic forms of Stemphylium occur on a wide range of host plants (Farr et al. 1989). Leaf blight caused by Stemphylium spp. (Basallote et al. 1993) has been described in many countries, including India (Raghayendra \& Pavgi 1975), South Africa (Aveling \& Naude 1992), Spain (Basallote et al. 1993), Australia (Suheri \& Price 2000), China (Zheng et al. 2009), England (Ruehle 1930), Malaysia (Nasehi et al. 2014) and Turkey (Polat et al. 2012). Stemphylium vesicarium has been reported in Spain, whereas $S$. solani is the most prevalent species recorded in China. Stemphylium vesicarium has also been recorded from Po Valley in Italy causing brown spot on leaves and fruits of pear (Ponti et al. 1982). This disease was subsequently found in Spain and France in the late 1980s and first observations of brown spot in The Netherlands and Belgium were reported in the early 1990s (Llorente \& Montesinos 2006, Polfliet 2002). Stemphylium leaf spot has become a huge challenge for California growers to overcome in producing large volumes of high quality, defect-free spinach (Koike et al. 2001) as well in Arizona, Delaware, Florida, Maryland and Quebec (Mou et al. 2008).

\section{Molecular phylogeny (Table 1, Fig. 1)}

Camara et al. (2002) used rDNA-ITS and glyceraldehyde-3-phosphate dehydrogenase (GPD) sequences to confirm the monophyly of the genus Stemphylium. An extensive phylogenetic study of Pleospora species with Stemphylium asexual morphs (Inderbitzin et al. 2009) left many unnamed and potentially new Stemphylium species. According to the one fungus-one name concept in the International Code of Nomenclature for algae, fungi and plants (ICN, McNeill et al. 2012), name changes in this genus became necessary. Woudenberg et al. (2017) revised the genus and accepted 28 species based on combined analyses of the ITS, gapdh and cmdA gene regions. ITS, gapdh and cmdA sequence data were analyzed by using 145 strains retrieved from the GeneBank following Woudenberg et al. (2017) (Table 1). Fig. 1 shows the phylogenetic placement of pathogenic isolates of Stemphylium species isolated from different host substrates and localities.

Table 1 Isolates used in this study for the analysis of combined ITS, gapdh, cmdA sequence data and their GenBank accession numbers. Bold accession numbers from ex-type strains.

\begin{tabular}{lllll}
\hline Taxon & Strain no. & ITS & Gapdh & cmdA \\
\hline Alternaria alternate & GV14-634a1 & KU850502 & KU850649 & KU850790 \\
Stemphylium amaranthi & CBS 124650 & KU850503 & KU850650 & KU850791 \\
& CBS 124651 & KU850504 & KU850651 & KU850792 \\
& CBS 124746 & KU850505 & KU850652 & KU850793 \\
& CBS 124750 & KU850506 & KU850653 & KU850794 \\
& CBS 124753 & KU850507 & KU850654 & KU850795 \\
& CBS 124984 & KU850508 & KU850655 & KU850796 \\
& CBS 124985 & KU850509 & KU850656 & KU850797 \\
& CBS 136589 & KU850510 & KU850657 & KU850798 \\
& CBS 338.73 & KU850511 & KU850658 & KU850799 \\
Stemphylium astragali & CBS 116583 & KU850512 & KU850659 & KU850800 \\
& CBS 378.54 & KU850513 & KU850660 & KU850801 \\
& CBS 116599 & KU850514 & KU850661 & KU850802 \\
& CBS 133512 & KU850515 & KU850662 & KU850803 \\
& & & & \\
\hline
\end{tabular}


Table 1 Continued.

\begin{tabular}{|c|c|c|c|c|}
\hline Taxon & Strain no. & ITS & gapdh & cmdA \\
\hline & CBS 133892 & KU850516 & KU850663 & KU850804 \\
\hline & CBS 136590 & KU850517 & KU850664 & KU850805 \\
\hline & CBS 136699 & KU850518 & KU850665 & KU850806 \\
\hline & CBS 137492 & KU850519 & KU850666 & KU850807 \\
\hline & CBS 141024 & KU850520 & KU850667 & KU850808 \\
\hline & CBS 141025 & KU850521 & KU850668 & KU850809 \\
\hline & CBS 141026 & KU850522 & KU850669 & KU850810 \\
\hline & GV11-196a1-3 & KU850523 & KU850670 & KU850811 \\
\hline & GV12-275a1 & KU850524 & KU850671 & KU850812 \\
\hline & GV12-276a1 & KU850525 & KU850672 & KU850813 \\
\hline & GV12-287a1 & KU850526 & KU850673 & KU850814 \\
\hline & GV12-336a1 & KU850527 & KU850674 & KU850815 \\
\hline & GV12-356a1 & KU850528 & KU850675 & KU850816 \\
\hline & GV12-367a1 & KU850529 & KU850676 & KU850817 \\
\hline & GV12-368a1 & KU850530 & KU850677 & KU850818 \\
\hline & GV12-403a1 & KU850531 & KU850678 & KU850819 \\
\hline & GV13-425a1 & KU850532 & KU850679 & KU850820 \\
\hline & GV13-436c2 & KU850533 & KU850680 & KU850821 \\
\hline & GV14-693a1 & KU850534 & KU850681 & KU850822 \\
\hline & IFZ2013-024 & KU850535 & KU850682 & KU850823 \\
\hline & IFZ2013-035 & KU850536 & KU850683 & KU850824 \\
\hline & IFZ2014-020 & KU850537 & KU850684 & KU850825 \\
\hline \multirow[t]{2}{*}{ Stemphylium botryosum } & CBS 714.68 & KC584238 & AF443881 & KU850826 \\
\hline & CBS 116596 & KU850538 & KU850685 & KU850827 \\
\hline Stemphylium callistephi & CBS 527.50 & KU850539 & KU850686 & KU850828 \\
\hline \multirow[t]{2}{*}{ Stemphylium canadense } & CBS 116602 & KU850641 & KU850782 & KU850932 \\
\hline & CBS 118081 & KU850642 & KU850783 & KU850933 \\
\hline Stemphylium chrysanthemicola & CBS 117255 & KU850640 & KU850781 & KU850931 \\
\hline Stemphylium drummondii & CBS 346.83 & GQ395365 & KU850687 & KU850829 \\
\hline \multirow[t]{7}{*}{ Stemphylium eturmiunum } & CBS 668.80 & KU850540 & KU850688 & KU850830 \\
\hline & CBS 109845 & KU850541 & KU850689 & KU850831 \\
\hline & CBS 122124 & KU850542 & KU850690 & KU850832 \\
\hline & CBS 122641 & KU850543 & KU850691 & KU850833 \\
\hline & CBS 124652 & KU850544 & KU850692 & KU850834 \\
\hline & CBS 133528 & KU850545 & KU850693 & KU850835 \\
\hline & CBS 138495 & KU850546 & KU850694 & KU850836 \\
\hline \multirow[t]{6}{*}{ Stemphylium gracilariae } & CBS 308.36 & KU850547 & KU850695 & KU850837 \\
\hline & CBS 273.55 & KU850548 & KU850696 & KU850838 \\
\hline & CBS 482.90 & KU850549 & AF443883 & KU850839 \\
\hline & CBS 115179 & KU850550 & KU850697 & KU850840 \\
\hline & CBS 115180 & KU850551 & KU850698 & KU850841 \\
\hline & CBS 125060 & KU850552 & KU850699 & KU850842 \\
\hline \multirow[t]{2}{*}{ Stemphylium halophilum } & CBS 337.73 & KU850553 & KU850700 & KU850843 \\
\hline & CBS 410.73 & KU850554 & KU850701 & KU850844 \\
\hline
\end{tabular}


Table 1 Continued.

\begin{tabular}{|c|c|c|c|c|}
\hline Taxon & Strain no. & ITS & gapdh & cmdA \\
\hline Stemphylium ixeridis & CBS 124748 & KU850590 & KU850737 & KU850881 \\
\hline \multirow{3}{*}{ Stemphylium lancipes } & CBS 101217 & KU850594 & KU850741 & KU850885 \\
\hline & CBS 116584 & KU850595 & AF443886 & KU850886 \\
\hline & CBS 133314 & KU850596 & KU850742 & KU850887 \\
\hline Stemphylium loti & CBS 407.54 & KU850597 & KU850743 & KU850888 \\
\hline Stemphylium lucomagnoense & CBS 116601 & KU850629 & KU850770 & KU850920 \\
\hline \multirow[t]{5}{*}{ Stemphylium lycii } & CBS 115192 & KU850598 & KU850744 & KU850889 \\
\hline & CBS 116582 & KU850599 & KU850745 & KU850890 \\
\hline & CBS 124982 & KU850600 & KU850746 & KU850891 \\
\hline & CBS 125240 & KU850601 & KU850747 & KU850892 \\
\hline & CBS 125241 & KU850602 & KU850748 & KU850893 \\
\hline \multirow[t]{15}{*}{ Stemphylium lycopersici } & CBS 333.73 & KU850603 & KU850749 & KU850894 \\
\hline & CBS 436.76 & KU850604 & KU850750 & KU850895 \\
\hline & CBS 463.78 & KU850605 & KU850751 & KU850896 \\
\hline & CBS 321.87 & KU850606 & KU850752 & KU850897 \\
\hline & CBS 116585 & KU850607 & AY317010 & KU850898 \\
\hline & CBS 116587 & KU850608 & KU850753 & KU850899 \\
\hline & CBS 120325 & KU850609 & KU850754 & KU850900 \\
\hline & CBS 120326 & KU850610 & KU850755 & KU850901 \\
\hline & CBS 122639 & KU850611 & KU850756 & KU850902 \\
\hline & CBS 122803 & KU850612 & KU850757 & KU850903 \\
\hline & CBS 123008 & KU850613 & KU850758 & KU850904 \\
\hline & CBS 124980 & KU850614 & KU850759 & KU850905 \\
\hline & CBS 124981 & KU850615 & KU850760 & KU850906 \\
\hline & CBS 124983 & KU850616 & KU850761 & KU850907 \\
\hline & CBS 135778 & KU850617 & AY317026 & KU850908 \\
\hline Stemphylium majusculum & CBS 717.68 & KU850618 & AF443891 & KU850909 \\
\hline \multirow[t]{2}{*}{ Stemphylium novae-zelandiae } & CBS 138157 & KU850630 & KU850771 & KU850921 \\
\hline & CBS 138295 & KU850631 & KU850772 & KU850922 \\
\hline Stemphylium paludiscirpi & CBS 109842 & KU850620 & KU850762 & KU850911 \\
\hline \multirow[t]{8}{*}{ Stemphylium sarciniforme } & CBS 335.33 & KU850621 & KU850763 & KU850912 \\
\hline & CBS 364.49 & KU850622 & KU850764 & KU850913 \\
\hline & CBS 110049 & KU850591 & KU850738 & KU850882 \\
\hline & CBS 116579 & KU850623 & AF443892 & KU850914 \\
\hline & CBS 116581 & KU850592 & KU850739 & KU850883 \\
\hline & CBS 133723 & KU850624 & KU850765 & KU850915 \\
\hline & CBS 136810 & KU850593 & KU850740 & KU850884 \\
\hline & CBS 138345 & KU850625 & KU850766 & KU850916 \\
\hline \multirow[t]{5}{*}{ Stemphylium simmonsii } & CBS 716.68 & KU850632 & KU850773 & KU850923 \\
\hline & CBS 116598 & KU850633 & KU850774 & KU850924 \\
\hline & CBS 116603 & KU850634 & KU850775 & KU850925 \\
\hline & CBS 116604 & KU850635 & KU850776 & KU850926 \\
\hline & CBS 133515 & KU850636 & KU850777 & KU850927 \\
\hline
\end{tabular}


Table 1 Continued.

\begin{tabular}{|c|c|c|c|c|}
\hline Taxon & Strain no. & ITS & gapdh & cmdA \\
\hline & CBS 133518 & KU850637 & KU850778 & KU850928 \\
\hline & CBS 133894 & KU850638 & KU850779 & KU850929 \\
\hline & CBS 134496 & KU850639 & KU850780 & KU850930 \\
\hline \multirow[t]{3}{*}{ Stemphylium solani } & CBS 408.54 & KU850626 & KU850767 & KU850917 \\
\hline & CBS 116586 & KU850627 & KU850768 & KU850918 \\
\hline & CBS 118082 & KU850628 & KU850769 & KU850919 \\
\hline \multirow[t]{4}{*}{ Stemphylium symphyti } & CBS 115268 & KU850643 & KU850784 & KU850934 \\
\hline & CBS 118796 & KU850644 & KU850785 & KU850935 \\
\hline & CBS 138069 & KU850645 & KU850786 & KU850936 \\
\hline & CBS 138070 & KU850646 & KU850787 & KU850937 \\
\hline Stemphylium trifolii & CBS 116580 & KU850647 & KU850788 & KU850938 \\
\hline Stemphylium triglochinicola & CBS 718.68 & KU850648 & KU850789 & KU850939 \\
\hline \multirow[t]{32}{*}{ Stemphylium vesicarium } & CBS 155.24 & KU850555 & KU850702 & KU850845 \\
\hline & CBS 157.24 & KU850556 & KU850703 & KU850846 \\
\hline & CBS 184.25 & KU850557 & KU850704 & KU850847 \\
\hline & CBS 273.31 & KU850558 & KU850705 & KU850848 \\
\hline & CBS 274.31 & KU850559 & KU850706 & KU850849 \\
\hline & CBS 307.36 & KU850560 & KU850707 & KU850850 \\
\hline & CBS 156.45 & KU850561 & KU850708 & KU850851 \\
\hline & CBS 322.49 & KU850562 & KU850709 & KU850852 \\
\hline & CBS 370.51 & KU850563 & KU850710 & KU850853 \\
\hline & CBS 368.59 & KU850564 & KU850711 & KU850854 \\
\hline & CBS 715.68 & KU850565 & KU850712 & KU850855 \\
\hline & CBS 406.76 & KU850566 & KU850713 & KU850856 \\
\hline & CBS 205.82 & KU850567 & KU850714 & KU850857 \\
\hline & CBS 191.86 & KC584239 & AF443884 & KU850858 \\
\hline & CBS 192.86 & KU850568 & KU850715 & KU850859 \\
\hline & CBS 311.92 & KU850569 & KU850716 & KU850860 \\
\hline & CBS 486.92 & KU850570 & KU850717 & KU850861 \\
\hline & CBS 109843 & KU850571 & KU850718 & KU850862 \\
\hline & CBS 109844 & KU850572 & KU850719 & KU850863 \\
\hline & CBS 115182 & KU850573 & KU850720 & KU850864 \\
\hline & CBS 115204 & KU850574 & KU850721 & KU850865 \\
\hline & CBS 122640 & KU850575 & KU850722 & KU850866 \\
\hline & CBS 123005 & KU850576 & KU850723 & KU850867 \\
\hline & CBS 123803 & KU850577 & KU850724 & KU850868 \\
\hline & CBS 124279 & KU850578 & KU850725 & KU850869 \\
\hline & CBS 124747 & KU850579 & KU850726 & KU850870 \\
\hline & CBS 124749 & KU850580 & KU850727 & KU850871 \\
\hline & CBS 124751 & KU850581 & KU850728 & KU850872 \\
\hline & CBS 124752 & KU850582 & KU850729 & KU850873 \\
\hline & CBS 125242 & KU850583 & KU850730 & KU850874 \\
\hline & CBS 133474 & KU850584 & KU850731 & KU850875 \\
\hline & CBS 133737 & KU850585 & KU850732 & KU850876 \\
\hline
\end{tabular}




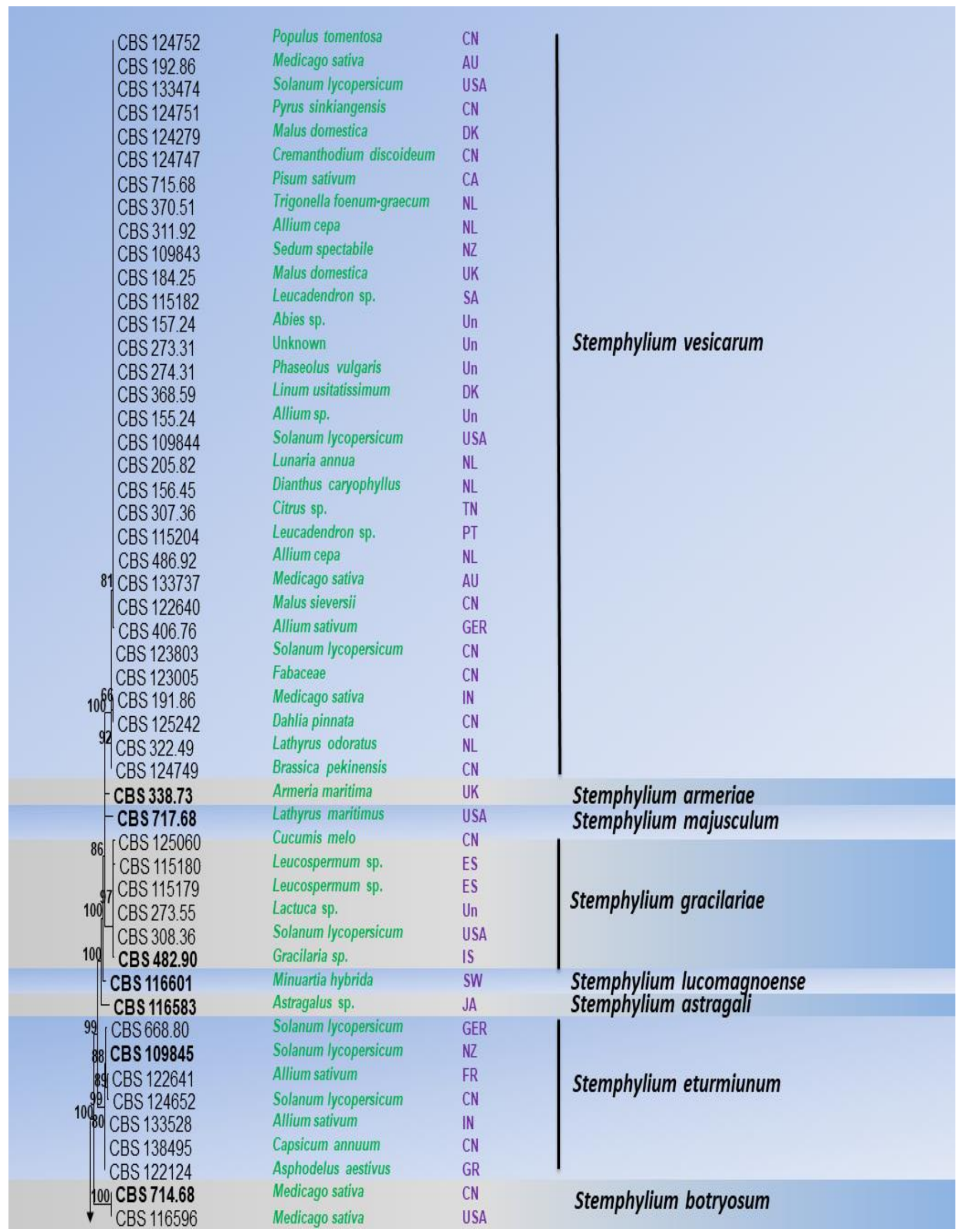




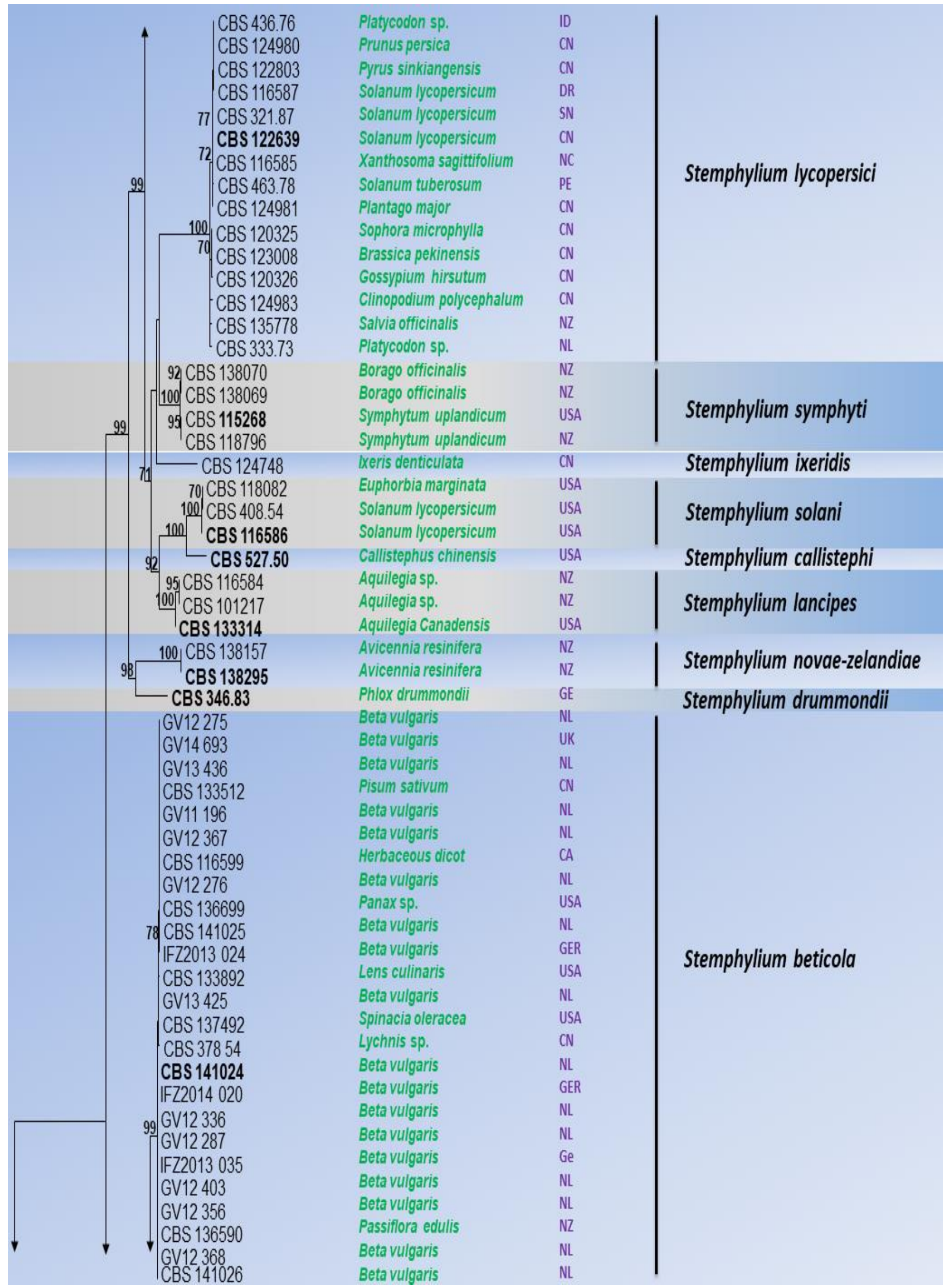




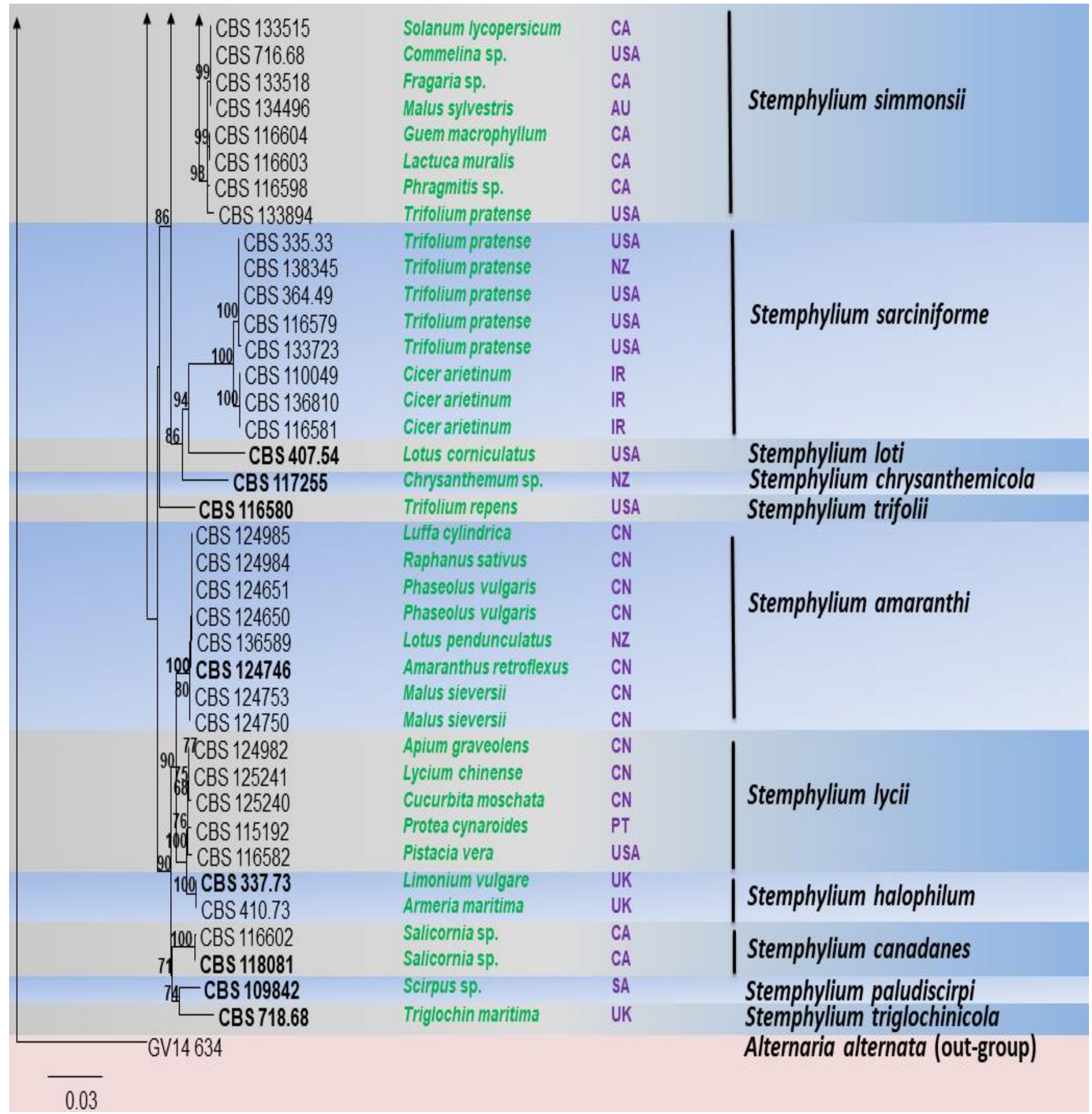

Fig. 1 - RAxML phylogenetic tree based on combined ITS, gapdh and cmdA sequence data from taxa of Stemphylium. Bootstrap support values for ML equal or greater than $65 \%$ are given above each branch. The tree is rooted to Alternaria alternata (GV14-634a1). AU: Australia; CN: China; DK: Denmark; DR: Dominican Republic; FR: France; GR: Greece; GER: Germany; IN: India; ID: Indonesia; IR: Iran; IS: Israel; JA: Japan; KA: Canada; NC: New Caledonia; NL: Netherlands; NZ: New Zealand; PE: Peru; PT: Portugal; SA: South Africa; ES: Spain; SN: Senegal; SW: Switzerland; TN: Tunisia; UK: United Kingdom; Un: Unknown; USA: America.

\section{Acknowledgments}

This work was funded by grants of the Beijing Science and Technology project D17110001617002, Thailand Research Fund (project No. TRG5880152), and the Mushroom Research Foundation provided partial support. Rashika Brahmanage offers his deepest gratitude to Monika C. Dayarathne for her helpful comments and advice. 


\section{References}

Ariyawansa HA, Thambugala KM, Manamgoda DS, Jayawardena R et al. 2015 - Towards a natural classification and backbone tree for Pleosporaceae. Fungal Diversity 71, 85-139.

Aveling TA, Naude SP. 1992 - First report of Stemphylium vesicarium on garlic in South Africa. Plant Disease 76(4), 426.

Aveling TAS, Snyman HG. 1993 - Infection studies of Stemphylium vesicarium on onion leaves. Mycological Research 97, 984-988.

Basallote MJ, Prados AM, Pe'rez de Algaba A, Melero JM. 1993 - First report in Spain of two leaf spots of garlic caused by Stemphylium vesicarium. Plant Disease 77, 952.

Basallote-Ureba MJ, Prados AM, Melero-Vara JM. 1998 - Effectiveness of tebuconazole and procymidone in the control of Stemphylium leaf spots in garlic. Crop Protection 17, 491-495.

Basallote-Ureba, MJ, Prados-Ligero AM, Melero-Vara JM. 1999 - Aetiology of leaf spot on garlic and onion caused by Stemphylium vesicarium in Spain. Plant Pathology 48, 139-145.

Bayaa B, Erskine W. 1998 - Lentil pathology. In: Allen D, Lenné J (eds). Pathology of Food and Pasture Legumes, Commonwealth Agricultural Bureaux International, U.K in association with International Crop Research Center for the Semi-Arid Tropics, Patancheru 502324. Andhra Pradesh, India, pp. 423-472.

Berg CC, Leath KT. 1996 - Responses of red clover cultivars to Stemphylium leaf spot. Crop Science 36(1), 71-74.

Camara MPS, O'Neill NR, van Berkum P. 2002 - Phylogeny of Stemphylium spp. based on ITS and glyceraldehyde-3-phosphate dehydrogenase gene sequences. Mycologia 94(4), 660672.

Crous PW, Groenewald JZ. 2017 - The genera of Fungi - G 4: Camarosporium and Dothiora. IMA Fungus 8, 131-152.

Crous PW, Wingfield MJ, Richardson DM, et al. 2016 - Fungal Planet description sheets: 400-468. Persoonia 36, 316-458.

De Gruyter J, Aveskamp MM, Woudenberg JHC, Verkley GJ et al. 2009 - Molecular phylogeny of Phoma and allied anamorph genera: towards a reclassification of the Phoma complex. Mycological Research 113, 508-519.

Ellis MB, Gibson IAS. 1975 - Stemphylium solani. C.M.I. Descriptions of Pathogenic Fungi and Bacteria no. 472, 1-2.

Enjoji S. 1931 - Two diseases of tomato (2) (in Japanese). Journal of Plant Protection Research 18, $48-53$.

Falloon PG, Falloon LM, Grogan RG. 1987 - Etiology and epidemiology of Stemphylium leaf spot and purple spot of asparagus in California. Phytopathology 77, 407-413.

Farr DF, Bills GF, Chamuris GP, Rossman AY. 1989 - Fungi on plants and plant products in the United States. St. Paul, Minnesota, APS Press. 1252 pp.

Gálvez L, Gil-Serna J, García M, Iglesias C, Palmero D. 2016 - Stemphylium leaf blight of garlic (Allium sativum) in Spain: taxonomy and in vitro fungicide response. The Plant Pathology Journal 32(5), 388-395.

Hanse B. 2013 - Research on Stemphylium spp. the causal agent of the yellow leaf spot disease in sugar beet in 2012. IRS, Bergen op Zoom, The Netherlands.

Hudson HJ. 1971 - The development of the saprophytic fungal flora as leaves senesce and fall. In: Preece TF, Dickinson CH (eds). Ecology of leaf surface microorganisms. London, Academic, pp. 447-455.

Ichikawa K, Sato T. 1994 - Leaf and stem spot of aster caused by Stemphylium vesicarium (in Japanese with English summary). Annals of the Phytopathological Society of Japan 60, 523526.

Inderbitzin P, Mehta YR, Berbee ML. 2009 - Pleospora species with Stemphylium anamorphs: a four locus phylogeny resolves new lineages yet does not distinguish among species in the Pleospora herbarum clade. Mycologia 101, 329-339. 
Index Fungorum. 2018 - http://www.indexfungorum.org/names/names.asp (accessed January 2018)

Irwin JAG. 1984 - Etiology of a new Stemphylium-incited leaf disease of alfalfa in Australia. Plant Disease 68, 531-532.

Johnson DA, Lunden JD. 1986 - Effects of wounding and wetting duration on infection of asparagus by Stemphylium vesicarium. Plant Disease 70, 419-420.

Köhl J, Groenenboom-De Haan B, de Geijn GV, Speksnijder A et al. 2009 - Pathogenicity of Stemphylium vesicarium from different hosts causing brown spot in pear. European Journal of Plant Pathology 124, 151-162.

Koike ST, Henderson DM, Butler EE. 2001 - Leaf spot disease of spinach in California caused by Stemphylium botryosum. Plant disease 85(2), 126-130.

Koike ST, Henderson DM, Butler EE. 2001 - Leaf spot disease of spinach in California caused by Stemphylium botryosum. Plant Disease 85, 126-130.

Koike ST, O’Neill N, Wolf J, Van Berkum P. Daugovish O. 2013 - Stemphylium leaf spot of parsley in California caused by Stemphylium vesicarium. Plant Disease 97, 315-322.

Kurose D, Kanegae Y, Misawa T, Ebihara Y et al. 2015 - Yellow spot of white lace flower caused by Pleospora herbarum in Japan. Japanese Journal of Phytopathology 81, 169-172.

Lamprecht SC, Baxter A, Thompson AH. 1984 - Stemphylium vesicarium on Medicago spp. in South Africa. Phytophylactica 16, 73-75.

Leuprecht B. 1990 - Stemphylium botryosum Wallr. on asparagus. Gesunde Pflanzen 42, 187-191.

Llorente I, Montesinos E. 2006 - Brown spot of pear: an emerging disease of economic importance in Europe. Plant Disease 90, 1368-1375.

Mou B, Koike ST, Du Toit LJ. 2008 - Screening for resistance to leaf spot diseases of spinach. HortScience, 43(6), 1706-1710.

McNeill J, Barrie FR, Buck WR, Demoulin V et al. 2012 - International Code of Nomenclature for algae, fungi, and plants (Melbourne code). Koeltz Scientific Books, Königstein, Germany [Regnum vegetabile no. 154.]

Mehta YR. 2001 - Genetic diversity among isolates of Stemphylium solani from cotton. Fitopatologia brasileira 26(4), 703-709.

Mentha YR. 1998 - Severe outbreak of Stemphylium leaf blight, a new disease of cotton in Brazil. Plant Disease 82, 333-336.

Miller ME, Taber RA, Amador JM. 1978 - Stemphylium blight of onion in South Texas. Plant Disease Reporter 62, 851-853.

Misawa T. 2009 - Brown leaf blight of Chinese chive caused by Stemphylium botryosum Wallroth. (Abstract in Japanese). Japanese Journal of Phytopathology 75, 87.

Nasehi A, Kadir JB, Esfahani MN, Mahmodi F et al. 2013 - An outbreak of leaf spot caused by Stemphylium solani on eggplant in Malaysia. Plant Disease 97, 689.

Nasehi A, Kadir JB, Nasr-Esfahani M, Abed-Ashtiani F et al. 2014 - Analysis of genetic and virulence variability of Stemphylium lycopersici associated with leaf spot of vegetable crops. European Journal of Plant Pathology 140(2), 261-273.

Polat Z, Besirli G, Sönmez I, Yavuz B. 2012 - First report of Stemphylium leaf blight of garlic (Allium sativum) caused by Stemphylium vesicarium in Turkey. New Disease Reports 25, 29.

Polfliet M. 2002 - Aantasting Stemphylium neemt met het jaar toe [Infection of Stemphylium increases every year]. Fruitteelt 92(20), 16-17.

Ponti I, Cavanni P, Brunelli A. 1982 - Maculatura bruna delle pere: eziologia e difesa. Informatore Fitopatologico 32, 35-40.

Prados-Ligero AM, González-Andújar JL, Melero-Vara JM, Basallote-Ureba MJ. 1998 Development of Pleospora allii on garlic debris infected by Stemphylium vesicarium. European Journal of Plant Protection 104, 861-870.

Raghayendra NN, Pavgi MS. 1975 - Stemphylium leaf blight of onion. Mycopathologia 56, 113118.

Reis A, Boiteux LS, Fonseca MN. 2011 - Identification of solanaceous and nonsolanaceous species as hosts of Stemphylium solani isolates in Brazil. Phytopathology 101, S152. 
Rossman AY, Crous PW, Hyde KD, Hawksworth DL et al. 2015 - Recommended names for pleomorphic genera in Dothideomycetes. IMA Fungus 6, 507-523.

Ruehle GD. 1930 - Cladosporium species from apple fruit and the perfect stage of Cladosporium herbarum Lk. Phytopathology 20(10), 854-807.

Seaney RR. 1973 - Birdsfoot trefoil. In: Heath ME, Metcalfe DS, Barnes RF (eds). Forages the science of grassland agriculture. Ames, Iowa, The Iowa State University Press. pp. 177-188.

Shibata S, Horiuchi S, Satou M, Yamauchi N. 2000 - Stemphylium vesicarium, another causal agent of leaf blight of welsh onion in Japan (in Japanese with English summary). Annual Report of the Society of Plant Protection of North Japan 51, 62-65.

Simmons EG. 1985 - Perfect states of Stemphylium II. Sydowia 38, 284-293.

Simmons EG. 1990 - Stemphylium leaf spot. Causal organisms. In: Stuteville DL, Erwin DC (eds). Compendium of alfalfa diseases, 2nd ed. St. Paul, Minnesota: APS Press. 84 pp.

Subedi S, Shrestha SM, Bahadur G, Thapa RB et al 2014 - Integrated Approach for the Management of New Threat Stemphylium botryosum walr Causing Blight of Lentil (Lens culinaris Medik). Türk Tarım ve Doğa Bilimleri, 6(6), 1209-1220.

Suheri H, Price TV. 2000 - Stemphylium leaf blight of garlic (Allium sativum) in Australia. Australasian Plant Pathology 29, 192-199.

Suzui T. 1973 - Stemphylium leaf spot (Stemphylium botryosum Wallr.) on asparagus plants (in Japanese with English summary). Annals of the Phytopathological Society of Japan 39, 364 366.

Tomioka K, Sato T, Sasaya T, Koganezawa H. 1997 - Leaf spot of kalanchoe caused by Stemphylium lycopersici. Annals of the Phytopathological Society of Japan 63, 337-340.

Tomioka K, Sato T. 2011 - Fruit rot of sweet pepper caused by Stemphylium lycopersici in Japan. Journal of General Plant Pathology 77, 342-344.

Vakalounakis DJ, Markakis EA. 2013 - First report of Stemphylium solani as the causal agent of a leaf spot on greenhouse cucumber. Plant Disease 97, 287-288.

Wallroth CFW. 1833 - Flora cryptogamica Germaniae section 2. J.L. Schrag, Nürnberg, Germany.

Wang Y, Zhang XG. 2009 - Two new species of Stemphylium from Shandong, China. Nova Hedwigia 88, 199-203.

Woudenberg JHC, Hanse B, Van Leeuwen GCM, Groenewald JZ et al. 2017 - Stemphylium revisited. Studies in Mycology 87, 77-103.

Zheng L, Lv R, Hsiang T, Huang J. 2009 - Host range and phytotoxicity of Stemphylium solani, causing leaf blight of garlic (Allium sativum) in China. European Journal of Plant Pathology 124(1), 21-30.

Zheng L, Lv R, Huang J, Jiang D et al. 2010 - Integrated control of garlic leaf blight caused by Stemphylium solani in China. Canadian Journal of Plant Pathology 32, 135-145. 